\title{
Viscosity approximation methods for nonexpansive semigroups in CAT(0) spaces
}

Rabian Wangkeeree ${ }^{1,2^{*}}$ and Pakkapon Preechasilp ${ }^{1}$

\section{*Correspondence:}

rabianw@nu.ac.th

'Department of Mathematics,

Faculty of Science, Naresuan

University, Phitsanulok, 65000,

Thailand

${ }^{2}$ Centre of Excellence in

Mathematics (CHE), Si Ayutthaya

Road, Bangkok, 10400, Thailand

\begin{abstract}
In this paper, we study the strong convergence of Moudafi's viscosity approximation methods for approximating a common fixed point of a one-parameter continuous semigroup of nonexpansive mappings in CAT(0) spaces. We prove that the proposed iterative scheme converges strongly to a common fixed point of a one-parameter continuous semigroup of nonexpansive mappings which is also a unique solution of the variational inequality. The results presented in this paper extend and enrich the existing literature.
\end{abstract}

Keywords: viscosity approximation method; nonexpansive semigroup; variational inequality; CAT(0) space; common fixed point

\section{Introduction}

Let $(X, d)$ be a metric space. A geodesic path joining $x \in X$ to $y \in X$ (or, more briefly, a geodesic from $x$ to $y$ ) is a map $c$ from a closed interval $[0, l] \subset \mathbb{R}$ to $X$ such that $c(0)=x$, $c(l)=y$, and $d\left(c(t), c\left(t^{\prime}\right)\right)=\left|t-t^{\prime}\right|$ for all $t, t^{\prime} \in[0, l]$. In particular, $c$ is an isometry and $d(x, y)=l$. The image $\alpha$ of $c$ is called a geodesic (or metric) segment joining $x$ and $y$. When it is unique, this geodesic segment is denoted by $[x, y]$. The space $(X, d)$ is said to be a geodesic space if every two points of $X$ are joined by a geodesic, and $X$ is said to be uniquely geodesic if there is exactly one geodesic joining $x$ and $y$ for each $x, y \in X$. A subset $Y \subseteq X$ is said to be convex if $Y$ includes every geodesic segment joining any two of its points. A geodesic triangle $\triangle\left(x_{1}, x_{2}, x_{3}\right)$ in a geodesic metric space $(X, d)$ consists of three points $x_{1}, x_{2}, x_{3}$ in $X$ (the vertices of $\triangle$ ) and a geodesic segment between each pair of vertices (the edges of $\triangle)$. A comparison triangle for the geodesic triangle $\Delta\left(x_{1}, x_{2}, x_{3}\right)$ in $(X, d)$ is a triangle $\bar{\Delta}\left(x_{1}, x_{2}, x_{3}\right):=\Delta\left(\bar{x}_{1}, \bar{x}_{2}, \bar{x}_{3}\right)$ in the Euclidean plane $\mathbb{E}^{2}$ such that $d_{\mathbb{E}_{2}}\left(\bar{x}_{i}, \bar{x}_{j}\right)=d\left(x_{i}, x_{j}\right)$ for all $i, j \in\{1,2,3\}$.

A geodesic space is said to be a CAT(0) space if all geodesic triangles of appropriate size satisfy the following comparison axiom.

CAT(0): Let $\triangle$ be a geodesic triangle in $X$ and let $\bar{\Delta}$ be a comparison triangle for $\Delta$. Then $\triangle$ is said to satisfy the $\mathrm{CAT}(0)$ inequality if for all $x, y \in \Delta$ and all comparison points $\bar{x}, \bar{y} \in \bar{\Delta}$,

$$
d(x, y) \leq d_{\mathbb{E}^{2}}(\bar{x}, \bar{y}) .
$$

๑) 2013 Wangkeeree and Preechasilp; licensee Springer. This is an Open Access article distributed under the terms of the Creative Commons Attribution License (http://creativecommons.org/licenses/by/2.0), which permits unrestricted use, distribution, and reproduction in any medium, provided the original work is properly cited. 
If $x, y_{1}, y_{2}$ are points in a $\operatorname{CAT}(0)$ space and if $y_{0}$ is the midpoint of the segment $\left[y_{1}, y_{2}\right]$, then the $\mathrm{CAT}(0)$ inequality implies

$$
d^{2}\left(x, y_{0}\right) \leq \frac{1}{2} d^{2}\left(x, y_{1}\right)+\frac{1}{2} d^{2}\left(x, y_{2}\right)-\frac{1}{4} d^{2}\left(y_{1}, y_{2}\right) .
$$

This is the (CN)-inequality of Bruhat and Tits [1]. In fact ( $c f$. [2], p.163), a geodesic space is a $\operatorname{CAT}(0)$ space if and only if it satisfies the $(\mathrm{CN})$-inequality.

It is well known that any complete, simply connected Riemannian manifold having nonpositive sectional curvature is a $\mathrm{CAT}(0)$ space. Other examples include pre-Hilbert spaces, $\mathbb{R}$-trees (see [2]), Euclidean buildings (see [3]), the complex Hilbert ball with a hyperbolic metric (see [4]), and many others. Complete CAT(0) spaces are often called Hadamard spaces.

It is proved in [2] that a normed linear space satisfies the $(\mathrm{CN})$-inequality if and only if it satisfies the parallelogram identity, i.e., is a pre-Hilbert space; hence it is not so unusual to have an inner product-like notion in Hadamard spaces. Berg and Nikolaev [5] introduced the concept of quasilinearization as follows.

Let us formally denote a pair $(a, b) \in X \times X$ by $\overrightarrow{a b}$ and call it a vector. Then quasilinearization is defined as a map $\langle\cdot, \cdot\rangle:(X \times X) \times(X \times X) \rightarrow \mathbb{R}$ defined by

$$
\langle\overrightarrow{a b}, \overrightarrow{c d}\rangle=\frac{1}{2}\left(d^{2}(a, d)+d^{2}(b, c)-d^{2}(a, c)-d^{2}(b, d)\right) \quad(a, b, c, d \in X) .
$$

It is easily seen that $\langle\overrightarrow{a b}, \overrightarrow{c d}\rangle=\langle\overrightarrow{c d}, \overrightarrow{a b}\rangle,\langle\overrightarrow{a b}, \overrightarrow{c d}\rangle=-\langle\overrightarrow{b a}, \overrightarrow{c d}\rangle$ and $\langle\overrightarrow{a x}, \overrightarrow{c d}\rangle+\langle\overrightarrow{x b}, \overrightarrow{c d}\rangle=\langle\overrightarrow{a b}, \overrightarrow{c d}\rangle$ for all $a, b, c, d, x \in X$. We say that $X$ satisfies the Cauchy-Schwarz inequality if

$$
\langle\overrightarrow{a b}, \overrightarrow{c d}\rangle \leq d(a, b) d(c, d)
$$

for all $a, b, c, d \in X$. It is known [5, Corollary 3$]$ that a geodesically connected metric space is a $\mathrm{CAT}(0)$ space if and only if it satisfies the Cauchy-Schwarz inequality.

In 2010, Kakavandi and Amini [6] introduced the concept of a dual space for $\operatorname{CAT}(0)$ spaces as follows. Consider the map $\Theta: \mathbb{R} \times X \times X \rightarrow C(X)$ defined by

$$
\Theta(t, a, b)(x)=t\langle\overrightarrow{a b}, \overrightarrow{a x}\rangle,
$$

where $C(X)$ is the space of all continuous real-valued functions on $X$. Then the CauchySchwarz inequality implies that $\Theta(t, a, b)$ is a Lipschitz function with a Lipschitz seminorm $L(\Theta(t, a, b))=|t| d(a, b)$ for all $t \in \mathbb{R}$ and $a, b \in X$, where

$$
L(f)=\sup \left\{\frac{f(x)-f(y)}{d(x, y)}: x, y \in X, x \neq y\right\}
$$

is the Lipschitz semi-norm of the function $f: X \rightarrow \mathbb{R}$. Now, define the pseudometric $D$ on $\mathbb{R} \times X \times X$ by

$$
D((t, a, b),(s, c, d))=L(\Theta(t, a, b)-\Theta(s, c, d)) .
$$

Lemma 1.1 [6, Lemma 2.1] $D((t, a, b),(s, c, d))=0$ if and only if $t\langle\overrightarrow{a b}, \overrightarrow{x y}\rangle=s\langle\overrightarrow{c d}, \overrightarrow{x y}\rangle$ for all $x, y \in X$. 
For a complete $\operatorname{CAT}(0)$ space $(X, d)$, the pseudometric space $(\mathbb{R} \times X \times X, D)$ can be considered as a subspace of the pseudometric space $(\operatorname{Lip}(X, R), L)$ of all real-valued Lipschitz functions. Also, $D$ defines an equivalence relation on $\mathbb{R} \times X \times X$, where the equivalence class of $t \overrightarrow{a b}:=(t, a, b)$ is

$$
[t \overrightarrow{a b}]=\{\overrightarrow{s c d}: t\langle\overrightarrow{a b}, \overrightarrow{x y}\rangle=s\langle\overrightarrow{c d}, \overrightarrow{x y}\rangle \forall x, y \in X\}
$$

The set $X^{*}:=\{[\overrightarrow{t a b}]:(t, a, b) \in \mathbb{R} \times X \times X\}$ is a metric space with metric $D$, which is called the dual metric space of $(X, d)$.

Recently, Dehghan and Rooin [7] introduced the duality mapping in CAT(0) spaces and studied its relation with subdifferential, by using the concept of quasilinearization. Then they presented a characterization of metric projection in $\mathrm{CAT}(0)$ spaces as follows.

Theorem 1.2 [7, Theorem 2.4] Let $C$ be a nonempty convex subset of a complete CAT(0) space $X, x \in X$ and $u \in C$. Then

$$
u=P_{C} x \quad \text { if and only if } \quad\langle\overrightarrow{y u}, \overrightarrow{u x}\rangle \geq 0 \quad \text { for all } y \in C .
$$

From now on, let $\mathbb{N}$ be the set of positive integers, let $\mathbb{R}$ be the set of real numbers, and let $\mathbb{R}^{+}$be the set of nonnegative real numbers. Let $C$ be a nonempty, closed and convex subset of a complete CAT(0) space $X$. A family $\mathcal{S}:=\left\{T(t): t \in \mathbb{R}^{+}\right\}$of self-mappings of $C$ is called a one-parameter continuous semigroup of nonexpansive mappings if the following conditions hold:

(i) for each $t \in \mathbb{R}^{+}, T(t)$ is a nonexpansive mapping on $C$, i.e.,

$$
d(T(t) x, T(t) y) \leq d(x, y), \quad \forall x, y \in C ;
$$

(ii) $T(s+t)=T(t) \circ T(s)$ for all $t, s \in \mathbb{R}^{+}$;

(iii) for each $x \in X$, the mapping $T(\cdot) x$ from $\mathbb{R}^{+}$into $C$ is continuous.

A family $\mathcal{S}:=\left\{T(t): t \in \mathbb{R}^{+}\right\}$of mappings is called a one-parameter strongly continuous semigroup of nonexpansive mappings if conditions (i), (ii) and (iii) and the following condition are satisfied:

(iv) $T(0) x=x$ for all $x \in C$.

We shall denote by $\mathcal{F}$ the common fixed point set of $\mathcal{S}$, that is,

$$
\mathcal{F}:=F(\mathcal{S})=\left\{x \in C: T(t) x=x, t \in \mathbb{R}^{+}\right\}=\bigcap_{t \in \mathbb{R}^{+}} F(T(t)) .
$$

One classical way to study nonexpansive mappings is to use contractions to approximate nonexpansive mappings. More precisely, take $t \in(0,1)$ and define a contraction $T_{t}: C \rightarrow C$ by

$$
T_{t}=t u+(1-t) T x, \quad \forall x \in C,
$$

where $u \in C$ is an arbitrary fixed element. Banach's contraction mapping principle guarantees that $T_{t}$ has a unique fixed point $x_{t}$ in $C$. It is unclear, in general, what the behavior of $x_{t}$ is as $t \rightarrow 0$, even if $T$ has a fixed point. However, in the case of $T$ having a fixed point, 
Browder [8] proved that $x_{t}$ converges strongly to a fixed point of $T$ that is nearest to $u$ in the framework of Hilbert spaces. Reich [9] extended Browder's result to the setting of Banach spaces and proved, in a uniformly smooth Banach space, that $x_{t}$ converges strongly to a fixed point of $T$ and the limit defines the (unique) sunny nonexpansive retraction from $C$ onto $F(T)$.

Halpern [10] introduced the following explicit iterative scheme (1.5) for a nonexpansive mapping $T$ on a subset $C$ of a Hilbert space by taking any points $u, x_{1} \in C$ and defined the iterative sequence $\left\{x_{n}\right\}$ by

$$
x_{n+1}=\alpha_{n} u+\left(1-\alpha_{n}\right) T x_{n} .
$$

He proved that the sequence $\left\{x_{n}\right\}$ generated by (1.5) converges to a fixed point of $T$.

It is an interesting problem to extend the above (Browder's [8] and Halpern's [10]) results to the nonexpansive semigroup case. In [11], Shioji and Takahashi introduced the following implicit iteration in a Hilbert space:

$$
x_{n}=\alpha_{n} u+\left(1-\alpha_{n}\right) \frac{1}{t_{n}} \int_{0}^{t_{n}} T(s) x_{n} d s \text {, }
$$

where $C$ is a nonempty closed convex subset of a real Hilbert space $H, u \in C,\left\{\alpha_{n}\right\}$ is a sequence in $(0,1),\left\{t_{n}\right\}$ is a sequence of positive real numbers divergent to $\infty$. Under suitable conditions, they proved strong convergence of $\left\{x_{n}\right\}$ to a member of $\mathcal{F}$.

Later, Suzuki [12] was the first to introduce in a Hilbert space the following iteration process:

$$
x_{n}=\alpha_{n} u+\left(1-\alpha_{n}\right) T\left(t_{n}\right) x_{n}, \quad \forall n \geq 1,
$$

where $\{T(t): t \geq 0\}$ is a strongly continuous semigroup of nonexpansive mappings on $C$ such that $\mathcal{F} \neq \varnothing$ and $\left\{\alpha_{n}\right\}$ and $\left\{t_{n}\right\}$ are appropriate sequences of real numbers. He proved that $\left\{x_{n}\right\}$ generated by (1.7) converges strongly to the element of $\mathcal{F}$ nearest to $u$. Using Moudafi's viscosity approximation methods, Song and Xu [13] introduced the following iteration process:

$$
x_{n}=\alpha_{n} f\left(x_{n}\right)+\left(1-\alpha_{n}\right) T\left(t_{n}\right) x_{n}, \quad \forall n \geq 1,
$$

and

$$
x_{n+1}=\alpha_{n} f\left(x_{n}\right)+\left(1-\alpha_{n}\right) T\left(t_{n}\right) x_{n}, \quad \forall n \geq 1 .
$$

They proved that $\left\{x_{n}\right\}$ converges to the same point of $\mathcal{F}$ in a reflexive strictly Banach space with a uniformly Gâteaux differentiable norm.

In the similar way, Dhompongsa et al. [14] extended Browder's iteration to a strongly continuous semigroup of nonexpansive mappings $\{T(t): t \geq 0\}$ in a complete $\operatorname{CAT}(0)$ space $X$ as follows:

$$
x_{n}=\alpha_{n} x_{0} \oplus T\left(t_{n}\right) x_{n}, \quad \forall n \geq 1,
$$


where $C$ is a nonempty closed convex subset of a complete CAT(0) space $X, x_{0} \in C$, $\left\{\alpha_{n}\right\}$ and $\left\{t_{n}\right\}$ are sequences of real numbers satisfying $0<\alpha_{n}<1, t_{n}>0$, and $\lim _{n \rightarrow \infty} t_{n}=$ $\lim _{n \rightarrow \infty} \alpha_{n} / t_{n}=0$. The proved that $\mathcal{F} \neq \emptyset$ and $\left\{x_{n}\right\}$ converges to the element of $\mathcal{F}$ nearest to $u$. For other related results, see $[15,16]$.

In 2012, Shi and Chen [17], studied the convergence theorems of the following Moudafi's viscosity iterations for a nonexpansive mapping $T$ : for a contraction $f$ on $C$ and $t \in(0,1)$, let $x_{t} \in C$ be a unique fixed point of the contraction $x \mapsto t f(x) \oplus(1-t) T x$; i.e.,

$$
x_{t}=t f\left(x_{t}\right) \oplus(1-t) T x_{t},
$$

and $x_{0} \in C$ is arbitrarily chosen and

$$
x_{n+1}=\alpha_{n} f\left(x_{n}\right) \oplus\left(1-\alpha_{n}\right) T x_{n}, \quad \forall n \geq 0,
$$

where $\left\{\alpha_{n}\right\} \subset(0,1)$. They proved $\left\{x_{t}\right\}$ defined by (1.10) converges strongly as $t \rightarrow 0$ to $\tilde{x} \in F(T)$ such that $\tilde{x}=P_{F(T)} f(\tilde{x})$ in the framework of $\mathrm{CAT}(0)$ space satisfying property $\mathcal{P}$, i.e., if for $x, u, y_{1}, y_{2} \in X$,

$$
d\left(x, P_{\left[x, y_{1}\right]} u\right) d\left(x, y_{1}\right) \leq d\left(x, P_{\left[x, y_{2}\right]} u\right) d\left(x, y_{2}\right)+d(x, u) d\left(y_{1}, y_{2}\right)
$$

Furthermore, they also obtained that $\left\{x_{n}\right\}$ defined by (1.11) converges strongly as $n \rightarrow \infty$ to $\tilde{x} \in F(T)$ under certain appropriate conditions imposed on $\left\{\alpha_{n}\right\}$.

By using the concept of quasilinearization, Wangkeeree and Preechasilp [18] improved Shi and Chen's results. In fact, they proved the strong convergence theorems for two given iterative schemes (1.10) and (1.11) in a complete CAT( 0 ) space without the property $\mathcal{P}$.

Motivated and inspired by Song and Xu [13], Dhompongsa et al. [14], and Wangkeeree and Preechasilp [18], in this paper we aim to study the strong convergence theorems of Moudafi's viscosity approximation methods for a one-parameter continuous semigroup of nonexpansive mappings $\mathcal{S}:=\left\{T(t): t \in \mathbb{R}^{+}\right\}$in $\mathrm{CAT}(0)$ spaces. Let $C$ be a nonempty, closed and convex subset of a CAT $(0)$ space $X$. For a given contraction $f$ on $C$ and $\alpha_{n} \in(0,1)$, let $x_{n} \in C$ be a unique fixed point of the contraction $x \mapsto \alpha_{n} f(x) \oplus\left(1-\alpha_{n}\right) T\left(t_{n}\right) x$; i.e.,

$$
x_{n}=\alpha_{n} f\left(x_{n}\right) \oplus\left(1-\alpha_{n}\right) T\left(t_{n}\right) x_{n}, \quad n \geq 0,
$$

and

$$
x_{n+1}=\alpha_{n} f\left(x_{n}\right) \oplus\left(1-\alpha_{n}\right) T\left(t_{n}\right) x_{n}, \quad n \geq 0 .
$$

We prove that the iterative schemes $\left\{x_{n}\right\}$ defined by (1.12) and $\left\{x_{n}\right\}$ defined by (1.13) converge strongly to the same point $\tilde{x}$ such that $\tilde{x}=P_{\mathcal{F}} f(\tilde{x})$, which is the unique solution of the variational inequality

$$
\langle\overrightarrow{\tilde{x} f} \tilde{x}, \vec{x} \tilde{x}\rangle \geq 0, \quad x \in \mathcal{F}
$$

where $\mathcal{F}$ is the common fixed point set of $\mathcal{S}$, that is,

$$
\mathcal{F}:=F(\mathcal{S})=\left\{x \in C: T(t) x=x, t \in \mathbb{R}^{+}\right\}=\bigcap_{t \in \mathbb{R}^{+}} F(T(t)) .
$$




\section{Preliminaries}

In this paper, we write $(1-t) x \oplus t y$ for the unique point $z$ in the geodesic segment joining from $x$ to $y$ such that

$$
d(z, x)=t d(x, y) \quad \text { and } \quad d(z, y)=(1-t) d(x, y) .
$$

We also denote by $[x, y]$ the geodesic segment joining from $x$ to $y$, that is, $[x, y]=\{(1-t) x \oplus$ ty: $t \in[0,1]\}$. A subset $C$ of a CAT(0) space is convex if $[x, y] \subseteq C$ for all $x, y \in C$.

The following lemmas play an important role in our paper.

Lemma 2.1 [2, Proposition 2.2] Let $X$ be a CAT(0) space, $p, q, r, s \in X$ and $\lambda \in[0,1]$. Then

$$
d(\lambda p \oplus(1-\lambda) q, \lambda r \oplus(1-\lambda) s) \leq \lambda d(p, r)+(1-\lambda) d(q, s) .
$$

Lemma $2.2[19$, Lemma 2.4] Let $X$ be a CAT(0) space, $x, y, z \in X$ and $\lambda \in[0,1]$. Then

$$
d(\lambda x \oplus(1-\lambda) y, z) \leq \lambda d(x, z)+(1-\lambda) d(y, z) .
$$

Lemma 2.3 [19, Lemma 2.5] Let $X$ be a CAT(0) space, $x, y, z \in X$ and $\lambda \in[0,1]$. Then

$$
d^{2}(\lambda x \oplus(1-\lambda) y, z) \leq \lambda d^{2}(x, z)+(1-\lambda) d^{2}(y, z)-\lambda(1-\lambda) d^{2}(x, y) .
$$

The concept of $\Delta$-convergence introduced by Lim [20] in 1976 was shown by Kirk and Panyanak [21] in CAT(0) spaces to be very similar to the weak convergence in Banach space setting. Next, we give the concept of $\Delta$-convergence and collect some basic properties.

Let $\left\{x_{n}\right\}$ be a bounded sequence in a $\operatorname{CAT}(0)$ space $X$. For $x \in X$, we set

$$
r\left(x,\left\{x_{n}\right\}\right)=\limsup _{n \rightarrow \infty} d\left(x, x_{n}\right)
$$

The asymptotic radius $r\left(\left\{x_{n}\right\}\right)$ of $\left\{x_{n}\right\}$ is given by

$$
r\left(\left\{x_{n}\right\}\right)=\inf \left\{r\left(x,\left\{x_{n}\right\}\right): x \in X\right\},
$$

and the asymptotic center $A\left(\left\{x_{n}\right\}\right)$ of $\left\{x_{n}\right\}$ is the set

$$
A\left(\left\{x_{n}\right\}\right)=\left\{x \in X: r\left(x,\left\{x_{n}\right\}\right)=r\left(\left\{x_{n}\right\}\right)\right\} .
$$

It is known from Proposition 7 of [22] that in a complete $\mathrm{CAT}(0)$ space, $A\left(\left\{x_{n}\right\}\right)$ consists of exactly one point. A sequence $\left\{x_{n}\right\} \subset X$ is said to $\Delta$-converge to $x \in X$ if $A\left(\left\{x_{n_{k}}\right\}\right)=$ $\{x\}$ for every subsequence $\left\{x_{n_{k}}\right\}$ of $\left\{x_{n}\right\}$. The uniqueness of an asymptotic center implies that a CAT(0) space $X$ satisfies Opial's property, i.e., for given $\left\{x_{n}\right\} \subset X$ such that $\left\{x_{n}\right\} \Delta$ converges to $x$ and given $y \in X$ with $y \neq x$,

$$
\limsup _{n \rightarrow \infty} d\left(x_{n}, x\right)<\limsup _{n \rightarrow \infty} d\left(x_{n}, y\right) .
$$

Since it is not possible to formulate the concept of demiclosedness in a CAT( 0$)$ setting, as stated in linear spaces, let us formally say that ' $I-T$ is demiclosed at zero' if the conditions $\left\{x_{n}\right\} \subseteq C \Delta$-converges to $x$ and $d\left(x_{n}, T x_{n}\right) \rightarrow 0$ imply $x \in F(T)$. 
Lemma 2.4 [21] Every bounded sequence in a complete CAT(0) space always has a $\Delta$ convergent subsequence.

Lemma 2.5 [23] If $C$ is a closed convex subset of a complete CAT(0) space and if $\left\{x_{n}\right\}$ is a bounded sequence in $C$, then the asymptotic center of $\left\{x_{n}\right\}$ is in $C$.

Lemma 2.6 [23] If $C$ is a closed convex subset of $X$ and $T: C \rightarrow X$ is a nonexpansive mapping, then the conditions $\left\{x_{n}\right\} \Delta$-converges to $x$ and $d\left(x_{n}, T x_{n}\right) \rightarrow 0$ imply $x \in C$ and $T x=x$.

Having the notion of quasilinearization, Kakavandi and Amini [6] introduced the following notion of convergence.

A sequence $\left\{x_{n}\right\}$ in the complete $\operatorname{CAT}(0)$ space $(X, d) w$-converges to $x \in X$ if

$$
\lim _{n \rightarrow \infty}\left\langle\overrightarrow{x x_{n}}, \overrightarrow{x y}\right\rangle=0
$$

i.e., $\lim _{n \rightarrow \infty}\left(d^{2}\left(x_{n}, x\right)-d^{2}\left(x_{n}, y\right)+d^{2}(x, y)\right)=0$ for all $y \in X$.

It is obvious that convergence in the metric implies $w$-convergence, and it is easy to check that $w$-convergence implies $\Delta$-convergence [6, Proposition 2.5], but it is showed in [24, Example 4.7] that the converse is not valid. However, the following lemma shows another characterization of $\Delta$-convergence as well as, more explicitly, a relation between $w$-convergence and $\Delta$-convergence.

Lemma 2.7 [24, Theorem 2.6] Let $X$ be a complete $\mathrm{CAT}(0)$ space, $\left\{x_{n}\right\}$ be a sequence in $X$ and $x \in X$. Then $\left\{x_{n}\right\} \Delta$-converges to $x$ if and only if $\lim \sup _{n \rightarrow \infty}\left\langle\overrightarrow{x x_{n}, \overrightarrow{x y}}\right\rangle \leq 0$ for all $y \in X$.

Lemma 2.8 [25, Lemma 2.1] Let $\left\{a_{n}\right\}$ be a sequence of non-negative real numbers satisfying the property

$$
a_{n+1} \leq\left(1-\alpha_{n}\right) a_{n}+\alpha_{n} \beta_{n}, \quad n \geq 0,
$$

where $\left\{\alpha_{n}\right\} \subseteq(0,1)$ and $\left\{\beta_{n}\right\} \subseteq \mathbb{R}$ such that

(i) $\sum_{n=0}^{\infty} \alpha_{n}=\infty$;

(ii) $\lim \sup _{n \rightarrow \infty} \beta_{n} \leq 0$ or $\sum_{n=0}^{\infty}\left|\alpha_{n} \beta_{n}\right|<\infty$.

Then $\left\{a_{n}\right\}$ converges to zero as $n \rightarrow \infty$.

\section{Viscosity approximation methods}

In this section, we present the strong convergence theorems of Moudafi's viscosity approximation methods for a one-parameter continuous semigroup of nonexpansive mappings $\mathcal{S}:=\left\{T(t): t \in \mathbb{R}^{+}\right\}$in $\mathrm{CAT}(0)$ spaces. Before proving main results, we need the following two vital lemmas.

Lemma 3.1 Let $X$ be a complete $\mathrm{CAT}(0)$ space. Then, for all $u, x, y \in X$, the following inequality holds:

$$
d^{2}(x, u) \leq d^{2}(y, u)+2\langle\overrightarrow{x y}, \overrightarrow{x u}\rangle .
$$


Proof Using (1.2), we have that

$$
\begin{aligned}
d^{2}(y, u)-d^{2}(x, u)-2\langle\overrightarrow{y x}, \overrightarrow{x u}\rangle & =d^{2}(y, u)-d^{2}(x, u)-2\langle\overrightarrow{y u}, \overrightarrow{x u}\rangle-2\langle\overrightarrow{u x}, \overrightarrow{x u}\rangle \\
& =d^{2}(y, u)-d^{2}(x, u)-2\langle\overrightarrow{y u}, \overrightarrow{x u}\rangle+2 d^{2}(x, u) \\
& =d^{2}(y, u)+d^{2}(x, u)-2\langle\overrightarrow{y u}, \overrightarrow{x u}\rangle \\
& \geq d^{2}(y, u)+d^{2}(x, u)-2 d(y, u) d(x, u) \\
& =\left(d^{2}(y, u)-d^{2}(x, u)\right)^{2} \geq 0 .
\end{aligned}
$$

Therefore we obtain that

$$
d^{2}(x, u) \leq d^{2}(y, u)+2\langle\overrightarrow{x y}, \overrightarrow{x u}\rangle,
$$

which is the desired result.

Lemma 3.2 Let $X$ be a $\mathrm{CAT}(0)$ space. For any $t \in[0,1]$ and $u, v \in X$, let $u_{t}=t u \oplus(1-t) v$. Then, for all $x, y \in X$,

(i) $\left\langle\overrightarrow{u_{t} x}, \overrightarrow{u_{t} y}\right\rangle \leq t\left\langle\overrightarrow{u_{x}}, \overrightarrow{u_{t} y}\right\rangle+(1-t)\left\langle\overrightarrow{v x}, \overrightarrow{u_{t} y}\right\rangle$;

(ii) $\left\langle\overrightarrow{u_{t} x}, \overrightarrow{u y}\right\rangle \leq t\langle\overrightarrow{u x}, \overrightarrow{u y}\rangle+(1-t)\langle\overrightarrow{v x}, \overrightarrow{u y}\rangle$ and $\left\langle\overrightarrow{u_{t} x}, \overrightarrow{v y}\right\rangle \leq t\langle\overrightarrow{u x}, \overrightarrow{v y}\rangle+(1-t)\langle\overrightarrow{v x}, \overrightarrow{v y}\rangle$.

Proof (i) It follows from (CN)-inequality (1.1) that

$$
\begin{aligned}
2\left\langle\overrightarrow{u_{t} x}, \overrightarrow{u_{t} y}\right\rangle= & d^{2}\left(u_{t}, y\right)+d^{2}\left(x, u_{t}\right)-d^{2}(x, y) \\
\leq & t d^{2}(u, y)+(1-t) d^{2}(v, y)-t(1-t) d^{2}(u, v)+d^{2}\left(x, u_{t}\right)-d^{2}(x, y) \\
= & t d^{2}(u, y)+t d^{2}\left(x, u_{t}\right)-t d^{2}\left(u, u_{t}\right)-t d^{2}(x, y) \\
& +(1-t) d^{2}(v, y)+(1-t) d^{2}\left(x, u_{t}\right)-(1-t) d^{2}\left(v, u_{t}\right)-(1-t) d^{2}(x, y) \\
& +t d^{2}\left(u, u_{t}\right)+(1-t) d^{2}\left(v, u_{t}\right)-t(1-t) d^{2}(u, v) \\
= & t\left[d^{2}(u, y)+d^{2}\left(x, u_{t}\right)-d^{2}\left(u, u_{t}\right)-d^{2}(x, y)\right] \\
& +(1-t)\left[d^{2}(v, y)+d^{2}\left(x, u_{t}\right)-d^{2}\left(v, u_{t}\right)-d^{2}(x, y)\right] \\
& +t(1-t)^{2} d^{2}(u, v)+(1-t) t^{2} d^{2}(u, v)-t(1-t) d^{2}(u, v) \\
= & t\left\langle\overrightarrow{u_{x} x,} \overrightarrow{u_{t} y}\right\rangle+(1-t)\left\langle\overrightarrow{v x}, \overrightarrow{u_{t} y}\right\rangle .
\end{aligned}
$$

(ii) The proof is similar to (i).

For any $\alpha_{n} \in(0,1), t_{n} \in[0, \infty)$ and a contraction $f$ with coefficient $\alpha \in(0,1)$, define the mapping $G_{n}: C \rightarrow C$ by

$$
G_{n}(x)=\alpha_{n} f(x) \oplus\left(1-\alpha_{n}\right) T\left(t_{n}\right) x, \quad \forall x \in C .
$$

It is not hard to see that $G_{n}$ is a contraction on $C$. Indeed, for $x, y \in C$, we have

$$
\begin{aligned}
d\left(G_{n}(x), G_{n}(y)\right) & =d\left(\alpha_{n} f(x) \oplus\left(1-\alpha_{n}\right) T\left(t_{n}\right) x, \alpha_{n} f(y) \oplus\left(1-\alpha_{n}\right) T\left(t_{n}\right) y\right) \\
& \leq d\left(\alpha_{n} f(x) \oplus\left(1-\alpha_{n}\right) T\left(t_{n}\right) x, \alpha_{n} f(y) \oplus\left(1-\alpha_{n}\right) T\left(t_{n}\right) x\right)
\end{aligned}
$$




$$
\begin{aligned}
& +d\left(\alpha_{n} f(y) \oplus\left(1-\alpha_{n}\right) T\left(t_{n}\right) x, \alpha_{n} f(y) \oplus\left(1-\alpha_{n}\right) T\left(t_{n}\right) y\right) \\
\leq & \alpha_{n} d(f(x), f(y))+\left(1-\alpha_{n}\right) d\left(T\left(t_{n}\right) x, T\left(t_{n}\right) y\right) \\
\leq & \alpha_{n} \alpha d(x, y)+\left(1-\alpha_{n}\right) d(x, y) \\
= & \left(1-\alpha_{n}(1-\alpha)\right) d(x, y) .
\end{aligned}
$$

Therefore we have that $G_{n}$ is a contraction mapping. Let $x_{n} \in C$ be the unique fixed point of $G_{n}$; that is,

$$
x_{n}=\alpha_{n} f\left(x_{n}\right) \oplus\left(1-\alpha_{n}\right) T\left(t_{n}\right) x_{n} \quad \text { for all } n \geq 0 .
$$

Now we are in a position to state and prove our main results.

Theorem 3.3 Let $C$ be a closed convex subset of a complete CAT(0) space $X$, and let $\{T(t)\}$ be a one-parameter continuous semigroup of nonexpansive mappings on $C$ satisfying $\mathcal{F} \neq \emptyset$ and uniformly asymptotically regular (in short, u.a.r.) on $C$, that is, for all $h \geq 0$ and any bounded subset $B$ of $C$,

$$
\lim _{t \rightarrow \infty} \sup _{x \in B} d(T(h)(T(t) x), T(t) x)=0 .
$$

Let $f$ be a contraction on $C$ with coefficient $0<\alpha<1$. Suppose that $t_{n} \in[0, \infty), \alpha_{n} \in(0,1)$ such that $\lim _{n \rightarrow \infty} t_{n}=\infty, \lim _{n \rightarrow \infty} \alpha_{n}=0$ and let $\left\{x_{n}\right\}$ be given by (3.2). Then $\left\{x_{n}\right\}$ converges strongly as $n \rightarrow \infty$ to $\tilde{x}$ such that $\tilde{x}=P_{\mathcal{F}} f(\tilde{x})$, which is equivalent to the following variational inequality:

$$
\langle\overrightarrow{\tilde{x} f} \tilde{x}, \vec{x} \tilde{x}\rangle \geq 0, \quad \forall x \in \mathcal{F}
$$

Proof We first show that $\left\{x_{n}\right\}$ is bounded. For any $p \in \mathcal{F}$, we have that

$$
\begin{aligned}
d\left(x_{n}, p\right) & =d\left(\alpha_{n} f\left(x_{n}\right) \oplus\left(1-\alpha_{n}\right) T\left(t_{n}\right) x_{n}, p\right) \leq \alpha_{n} d\left(f\left(x_{n}\right), p\right)+\left(1-\alpha_{n}\right) d\left(T\left(t_{n}\right) x_{n}, p\right) \\
& \leq \alpha_{n} d\left(f\left(x_{n}\right), p\right)+\left(1-\alpha_{n}\right) d\left(x_{n}, p\right) .
\end{aligned}
$$

Then

$$
d\left(x_{n}, p\right) \leq d\left(f\left(x_{n}\right), p\right) \leq d\left(f\left(x_{n}\right), f(p)\right)+d(f(p), p) \leq \alpha d\left(x_{n}, p\right)+d(f(p), p) .
$$

This implies that

$$
d\left(x_{n}, p\right) \leq \frac{1}{1-\alpha} d(f(p), p) .
$$

Hence $\left\{x_{n}\right\}$ is bounded, so are $\left\{T\left(t_{n}\right) x_{n}\right\}$ and $\left\{f\left(x_{n}\right)\right\}$. We get that

$$
\begin{aligned}
d\left(x_{n}, T\left(t_{n}\right) x_{n}\right) & =d\left(\alpha_{n} f\left(x_{n}\right) \oplus\left(1-\alpha_{n}\right) T\left(t_{n}\right) x_{n}, T\left(t_{n}\right) x_{n}\right) \\
& \leq \alpha_{n} d\left(f\left(x_{n}\right), T\left(t_{n}\right) x_{n}\right)+\left(1-\alpha_{n}\right) d\left(T\left(t_{n}\right) x_{n}, T\left(t_{n}\right) x_{n}\right) \\
& \leq \alpha_{n} d\left(f\left(x_{n}\right), T\left(t_{n}\right) x_{n}\right) \rightarrow 0 \quad \text { as } n \rightarrow \infty .
\end{aligned}
$$


Since $\{T(t)\}$ is u.a.r. and $\lim _{n \rightarrow \infty} t_{n}=\infty$, then for all $h>0$,

$$
\lim _{n \rightarrow \infty} d\left(T(h)\left(T\left(t_{n}\right) x_{n}\right), T\left(t_{n}\right) x_{n}\right) \leq \lim _{n \rightarrow \infty} \sup _{x \in B} d\left(T(h)\left(T\left(t_{n}\right) x\right), T\left(t_{n}\right) x\right)=0
$$

where $B$ is any bounded subset of $C$ containing $\left\{x_{n}\right\}$. Hence

$$
\begin{aligned}
d\left(x_{n}, T(h) x_{n}\right) \leq & d\left(x_{n}, T\left(t_{n}\right) x_{n}\right)+d\left(T\left(t_{n}\right) x_{n}, T(h)\left(T\left(t_{n}\right) x_{n}\right)\right) \\
& +d\left(T(h)\left(T\left(t_{n}\right) x_{n}\right), T(h) x_{n}\right) \\
\leq & 2 d\left(x_{n}, T\left(t_{n}\right) x_{n}\right)+d\left(T\left(t_{n}\right) x_{n}, T(h)\left(T\left(t_{n}\right) x_{n}\right)\right) \rightarrow 0 \quad \text { as } n \rightarrow \infty
\end{aligned}
$$

We will show that $\left\{x_{n}\right\}$ contains a subsequence converging strongly to $\tilde{x}$ such that $\tilde{x}=$ $P_{F(T)} f(\tilde{x})$, which is equivalent to the following variational inequality:

$$
\langle\overrightarrow{\tilde{x} f \tilde{x}}, \vec{x} \tilde{x}\rangle \geq 0, \quad x \in \mathcal{F}
$$

Since $\left\{x_{n}\right\}$ is bounded, by Lemma 2.4, there exists a subsequence $\left\{x_{n_{j}}\right\}$ of $\left\{x_{n}\right\}$ which $\Delta$-converges to a point $\tilde{x}$, denoted by $\left\{x_{j}\right\}$. We claim that $\tilde{x} \in \mathcal{F}$. Since every CAT(0) space has Opial's property, for any $h \geq 0$, if $T(h) \tilde{x} \neq \tilde{x}$, we have

$$
\begin{aligned}
\limsup _{j \rightarrow \infty} d\left(x_{j}, T(h) \tilde{x}\right) & \leq \limsup _{j \rightarrow \infty}\left\{d\left(x_{j}, T(h) x_{j}\right)+d\left(T(h) x_{j}, T(h) \tilde{x}\right)\right\} \\
& \leq \limsup _{j \rightarrow \infty}\left\{d\left(x_{j}, T(h) x_{j}\right)+d\left(x_{j}, \tilde{x}\right)\right\} \\
& =\limsup _{j \rightarrow \infty} d\left(x_{j}, \tilde{x}\right) \\
& <\limsup _{j \rightarrow \infty} d\left(x_{j}, T(h) \tilde{x}\right) .
\end{aligned}
$$

This is a contradiction, and hence $\tilde{x} \in \mathcal{F}$. So we have the claim. It follows from Lemma 3.2(i) that

$$
\begin{aligned}
& d^{2}\left(x_{j}, \tilde{x}\right)=\left\langle\overrightarrow{x_{j}} \vec{x}, \overrightarrow{x_{j}} \vec{x}\right\rangle \\
& \leq \alpha_{j}\left|\overrightarrow{f\left(x_{j}\right)} \overrightarrow{\tilde{x}}, \overrightarrow{x_{j}} \overrightarrow{\tilde{x}}\right\rangle+\left(1-\alpha_{j}\right)\left\langle\overrightarrow{T\left(t_{j}\right) x_{j} \tilde{x}, \overrightarrow{x_{j}} \vec{x}}\right\rangle \\
& \leq \alpha_{j}\left|\overrightarrow{f\left(x_{j}\right) \tilde{x}, \overrightarrow{x_{j}} \vec{x}}\right\rangle+\left(1-\alpha_{j}\right) d\left(T\left(t_{j}\right) x_{j}, \tilde{x}\right) d\left(x_{j}, \tilde{x}\right) \\
& \left.\leq \alpha_{j} \mid \overrightarrow{f\left(x_{j}\right) \tilde{x}}, \overrightarrow{x_{j}} \vec{x}\right)+\left(1-\alpha_{j}\right) d^{2}\left(x_{j}, \tilde{x}\right) .
\end{aligned}
$$

It follows that

$$
\begin{aligned}
d^{2}\left(x_{j}, \tilde{x}\right) & \leq\left\langle\overrightarrow{\left.f\left(x_{j}\right) \tilde{x}, \overrightarrow{x_{j}} \vec{x}\right\rangle}\right. \\
& =\left\langle\overrightarrow{f\left(x_{j}\right) f(\vec{x})}, \overrightarrow{x_{j}} \vec{x}\right\rangle+\left\langle\overrightarrow{\left.f(\tilde{x}) \tilde{x}, \overrightarrow{x_{j}} \vec{x}\right\rangle}\right. \\
& \leq d\left(f\left(x_{j}\right), f(\tilde{x})\right) d\left(x_{j}, \tilde{x}\right)+\left\langle\overrightarrow{f(\tilde{x}) \tilde{x}, \overrightarrow{x_{j}} \tilde{x}}\right\rangle \\
& \leq \alpha d^{2}\left(x_{j}, \tilde{x}\right)+\left\langle\overrightarrow{f(\tilde{x}) \tilde{x}}, \overrightarrow{x_{j}} \overrightarrow{\tilde{x}}\right\rangle
\end{aligned}
$$


and thus

$$
d^{2}\left(x_{j}, \tilde{x}\right) \leq \frac{1}{1-\alpha}\left|\overrightarrow{f(\tilde{x})} \vec{x}, \overrightarrow{x_{j}} \overrightarrow{\tilde{x}}\right| .
$$

Since $\left\{x_{j}\right\} \Delta$-converges to $\tilde{x}$, by Lemma 2.7 , we have

$$
\limsup _{n \rightarrow \infty}\left\langle\overrightarrow{f(\tilde{x}) \vec{x}}, \overrightarrow{x_{j}} \vec{x}\right\rangle \leq 0
$$

It follows from (3.6) that $\left\{x_{j}\right\}$ converges strongly to $\tilde{x}$. Next, we show that $\tilde{x}$ solves the variational inequality (3.3). Applying Lemma 2.3 , for any $q \in \mathcal{F}$,

$$
\begin{aligned}
d^{2}\left(x_{j}, q\right) & =d^{2}\left(\alpha_{j} f\left(x_{j}\right) \oplus\left(1-\alpha_{j}\right) T\left(t_{j}\right) x_{j}, q\right) \\
& \leq \alpha_{j} d^{2}\left(f\left(x_{j}\right), q\right)+\left(1-\alpha_{j}\right) d^{2}\left(T\left(t_{j}\right) x_{j}, q\right)-\alpha_{j}\left(1-\alpha_{j}\right) d^{2}\left(f\left(x_{j}\right), T\left(t_{j}\right) x_{j}\right) \\
& \leq \alpha_{j} d^{2}\left(f\left(x_{j}\right), q\right)+\left(1-\alpha_{j}\right) d^{2}\left(x_{j}, q\right)-\alpha_{j}\left(1-\alpha_{j}\right) d^{2}\left(f\left(x_{j}\right), T\left(t_{j}\right) x_{j}\right) .
\end{aligned}
$$

It implies that

$$
d^{2}\left(x_{j}, q\right) \leq d^{2}\left(f\left(x_{j}\right), q\right)-\left(1-\alpha_{j}\right) d^{2}\left(f\left(x_{j}\right), T\left(t_{j}\right) x_{j}\right) .
$$

Taking the limit through $j \rightarrow \infty$, we can get that

$$
d^{2}(\tilde{x}, q) \leq d^{2}(f(\tilde{x}), q)-d^{2}(f(\tilde{x}), \tilde{x}) .
$$

Hence

$$
0 \leq \frac{1}{2}\left[d^{2}(\tilde{x}, \tilde{x})+d^{2}(f(\tilde{x}), q)-d^{2}(\tilde{x}, q)-d^{2}(f(\tilde{x}), \tilde{x})\right]=\langle\overrightarrow{\tilde{x} f(\tilde{x})}, \overrightarrow{q \tilde{x}}\rangle, \quad \forall q \in \mathcal{F}
$$

That is, $\tilde{x}$ solves the inequality (3.3). Finally, we show that the sequence $\left\{x_{n}\right\}$ converges to $\tilde{x}$. Assume that $x_{n_{i}} \rightarrow \hat{x}$, where $i \rightarrow \infty$. By the same argument, we get that $\hat{x} \in \mathcal{F}$ and solves the variational inequality (3.3), i.e.,

$$
\langle\overrightarrow{\tilde{x} f \tilde{x}}, \overrightarrow{\tilde{x} \hat{x}}\rangle \leq 0
$$

and

$$
\langle\overrightarrow{\hat{x} f \hat{x}}, \vec{x} \tilde{x}\rangle \leq 0
$$

Adding up (3.7) and (3.8), we get that

$$
\begin{aligned}
0 & \geq\langle\overrightarrow{\tilde{x} f(\vec{x})}, \overrightarrow{\tilde{x} \hat{x}}\rangle-\langle\overrightarrow{\hat{x} f(\hat{x}),}, \overrightarrow{\tilde{x} \hat{x}}\rangle \\
& =\langle\overrightarrow{\tilde{x} f(\vec{x})}, \overrightarrow{\tilde{x} \hat{x}}\rangle+\langle\overrightarrow{f(\hat{x}) f(\vec{x})}, \overrightarrow{\tilde{x} \hat{x}}\rangle-\langle\overrightarrow{\hat{x} \tilde{x}}, \overrightarrow{\tilde{x} \hat{x}}\rangle-\langle\overrightarrow{\tilde{x} f(\vec{x})}, \overrightarrow{\tilde{x} \hat{x}}\rangle \\
& =\langle\overrightarrow{\tilde{x} \hat{x}}, \overrightarrow{\tilde{x} \hat{x}}\rangle-\langle\overrightarrow{f(\hat{x}) f(\tilde{x})}, \vec{x} \vec{x}\rangle \\
& \geq\langle\overrightarrow{\tilde{x} \hat{x}}, \overrightarrow{\tilde{x} \hat{x}}\rangle-d(f(\hat{x}), f(\tilde{x})) d(\hat{x}, \tilde{x})
\end{aligned}
$$




$$
\begin{aligned}
& \geq d^{2}(\tilde{x}, \hat{x})-\alpha d(\hat{x}, \tilde{x}) d(\hat{x}, \tilde{x}) \\
& \geq d^{2}(\tilde{x}, \hat{x})-\alpha d^{2}(\hat{x}, \tilde{x}) \\
& \geq(1-\alpha) d^{2}(\tilde{x}, \hat{x}) .
\end{aligned}
$$

Since $0<\alpha<1$, we have that $d(\tilde{x}, \hat{x})=0$, and so $\tilde{x}=\hat{x}$. Hence the sequence $x_{n}$ converges strongly to $\tilde{x}$, which is the unique solution to the variational inequality (3.3). This completes the proof.

$$
\text { If } f \equiv u \text {, then the following result can be obtained directly from Theorem 3.3. }
$$

Corollary 3.4 Let C be a closed convex subset of a complete CAT $(0)$ space $X$, and let $\{T(t)\}$ be a one-parameter continuous semigroup of nonexpansive mappings on $C$ satisfying $\mathcal{F} \neq \emptyset$ and uniformly asymptotically regular (in short, u.a.r.) on $C$, that is, for all $h \geq 0$ and any bounded subset $B$ of $C$,

$$
\lim _{t \rightarrow \infty} \sup _{x \in B} d(T(h)(T(t) x), T(t) x)=0 .
$$

Let $u$ be any element in C. Suppose $t_{n} \in[0, \infty), \alpha_{n} \in(0,1)$ such that $\lim _{n \rightarrow \infty} t_{n}=\infty$ and $\lim _{n \rightarrow \infty} \alpha_{n}=0$ and let $\left\{x_{n}\right\}$ be given by

$$
x_{n}=\alpha_{n} u \oplus\left(1-\alpha_{n}\right) T\left(t_{n}\right) x_{n} .
$$

Then $\left\{x_{n}\right\}$ converges strongly as $n \rightarrow \infty$ to $\tilde{x}$ such that $\tilde{x}=P_{\mathcal{F}} \tilde{x}$, which is equivalent to the following variational inequality:

$$
\langle\overrightarrow{\tilde{x} u}, \overrightarrow{x \tilde{x}}\rangle \geq 0, \quad x \in \mathcal{F} \text {. }
$$

Theorem 3.5 Let $C$ be a closed convex subset of a complete CAT(0) space $X$, and let $\{T(t)\}$ be a one-parameter continuous semigroup of nonexpansive mappings on $C$ satisfying $\mathcal{F} \neq \emptyset$ and uniformly asymptotically regular (in short, u.a.r.) on $C$, that is, for all $h \geq 0$ and any bounded subset $B$ of $C$,

$$
\lim _{t \rightarrow \infty} \sup _{x \in B} d(T(h)(T(t) x), T(t) x)=0 .
$$

Let $f$ be a contraction on $C$ with coefficient $0<\alpha<1$. Suppose that $t_{n} \in[0, \infty), \alpha_{n} \in(0,1)$, $x_{0} \in C$, and $\left\{x_{n}\right\}$ is given by

$$
x_{n+1}=\alpha_{n} f\left(x_{n}\right) \oplus\left(1-\alpha_{n}\right) T\left(t_{n}\right) x_{n}, \quad \forall n \geq 0,
$$

where $\left\{\alpha_{n}\right\} \subset(0,1)$ satisfies the following conditions:

(i) $\lim _{n \rightarrow \infty} \alpha_{n}=0$;

(ii) $\sum_{n=0}^{\infty} \alpha_{n}=\infty$ and

(iii) $\lim _{n \rightarrow \infty} t_{n}=\infty$.

Then $\left\{x_{n}\right\}$ converges strongly as $n \rightarrow \infty$ to $\tilde{x}$ such that $\tilde{x}=P_{\mathcal{F}} f(\tilde{x})$, which is equivalent to the variational inequality (3.3). 
Proof We first show that the sequence $\left\{x_{n}\right\}$ is bounded. For any $p \in \mathcal{F}$, we have that

$$
\begin{aligned}
d\left(x_{n+1}, p\right) & =d\left(\alpha_{n} f\left(x_{n}\right) \oplus\left(1-\alpha_{n}\right) T\left(t_{n}\right) x_{n}, p\right) \\
& \leq \alpha_{n} d\left(f\left(x_{n}\right), p\right)+\left(1-\alpha_{n}\right) d\left(T\left(t_{n}\right) x_{n}, p\right) \\
& \leq \alpha_{n}\left(d\left(f\left(x_{n}\right), f(p)\right)+d(f(p), p)\right)+\left(1-\alpha_{n}\right) d\left(T\left(t_{n}\right) x_{n}, p\right) \\
& \leq \max \left\{d\left(x_{n}, p\right), \frac{1}{1-\alpha} d(f(p), p)\right\} .
\end{aligned}
$$

By induction, we have

$$
d\left(x_{n}, p\right) \leq \max \left\{d\left(x_{0}, p\right), \frac{1}{1-\alpha} d(f(p), p)\right\}
$$

for all $n \in \mathbb{N}$. Hence $\left\{x_{n}\right\}$ is bounded, so are $\left\{T\left(t_{n}\right) x_{n}\right\}$ and $\left\{f\left(x_{n}\right)\right\}$. Using the assumption that $\lim _{n \rightarrow \infty} \alpha_{n}=0$, we get that

$$
d\left(x_{n+1}, T\left(t_{n}\right) x_{n}\right) \leq \alpha_{n} d\left(f\left(x_{n}\right), T\left(t_{n}\right) x_{n}\right) \rightarrow 0 \quad \text { as } n \rightarrow \infty .
$$

Since $\{T(t)\}$ is u.a.r. and $\lim _{n \rightarrow \infty} t_{n}=\infty$, then for all $h \geq 0$,

$$
\lim _{n \rightarrow \infty} d\left(T(h)\left(T\left(t_{n}\right) x_{n}\right), T\left(t_{n}\right) x_{n}\right) \leq \lim _{n \rightarrow \infty} \sup _{x \in B} d\left(T(h)\left(T\left(t_{n}\right) x\right), T\left(t_{n}\right) x\right)=0,
$$

where $B$ is any bounded subset of $C$ containing $\left\{x_{n}\right\}$. Hence

$$
\begin{aligned}
& d\left(x_{n+1}, T(h) x_{n+1}\right) \\
& \quad \leq d\left(x_{n+1}, T\left(t_{n}\right) x_{n}\right)+d\left(T\left(t_{n}\right) x_{n}, T(h)\left(T\left(t_{n}\right) x_{n}\right)\right) \\
& \quad+d\left(T(h)\left(T\left(t_{n}\right) x_{n}\right), T(h) x_{n+1}\right) \\
& \quad \leq 2 d\left(x_{n+1}, T\left(t_{n}\right) x_{n}\right)+d\left(T\left(t_{n}\right) x_{n}, T(h)\left(T\left(t_{n}\right) x_{n}\right)\right) \rightarrow 0 \quad \text { as } n \rightarrow \infty .
\end{aligned}
$$

Let $\left\{z_{m}\right\}$ be a sequence in $C$ such that

$$
z_{m}=\alpha_{m} f\left(z_{m}\right) \oplus\left(1-\alpha_{m}\right) T\left(t_{m}\right) z_{m} .
$$

It follows from Theorem 3.3 that $\left\{z_{m}\right\}$ converges strongly as $m \rightarrow \infty$ to a fixed point $\tilde{x} \in \mathcal{F}$, which solves the variational inequality (3.3). Now, we claim that

$$
\limsup _{n \rightarrow \infty}\left\langle\overrightarrow{f(\tilde{x}) \tilde{x}}, \overrightarrow{x_{n+1}} \overrightarrow{\tilde{x}}\right\rangle \leq 0
$$

It follows from Lemma 3.2(i) that

$$
\begin{aligned}
d^{2}\left(z_{m}, x_{n+1}\right) & =\left\langle\overrightarrow{z_{m} x_{n+1}}, \overrightarrow{z_{m} x_{n+1}}\right\rangle \\
& \leq \alpha_{m}\left|\overrightarrow{f\left(z_{m}\right) x_{n+1}}, \overrightarrow{z_{m} x_{n+1}}\right\rangle+\left(1-\alpha_{m}\right)\left|\overrightarrow{T\left(t_{m}\right) z_{m} x_{n+1}}, \overrightarrow{z_{m} x_{n+1}}\right\rangle \\
& =\alpha_{m}\left\langle\overrightarrow{f\left(z_{m}\right) f(\vec{x})}, \overrightarrow{z_{m} x_{n+1}}\right\rangle+\alpha_{m}\left\langle\overrightarrow{f(\tilde{x}) \tilde{x}}, \overrightarrow{z_{m} x_{n+1}}\right\rangle+\alpha_{m}\left\langle\overrightarrow{\tilde{x} z_{m}}, \overrightarrow{z_{m} x_{n+1}}\right\rangle
\end{aligned}
$$




$$
\begin{aligned}
& +\alpha_{m}\left\langle\overrightarrow{z_{m} x_{n+1}}, \overrightarrow{z_{m} x_{n+1}}\right\rangle+\left(1-\alpha_{m}\right)\left|\overrightarrow{T\left(t_{m}\right) z_{m} T\left(t_{m}\right) x_{n+1}}, \overrightarrow{z_{m} x_{n+1}}\right\rangle \\
& +\left(1-\alpha_{m}\right)\left\langle\overrightarrow{T\left(t_{m}\right) x_{n+1} x_{n+1}}, \overrightarrow{z_{m} x_{n+1}}\right\rangle \\
\leq & \alpha_{m} \alpha d\left(z_{m}, \tilde{x}\right) d\left(z_{m}, x_{n+1}\right)+\alpha_{m}\left\langle\overrightarrow{f(\tilde{x}) \tilde{x}}, \overrightarrow{z_{m} x_{n+1}}\right\rangle+\alpha_{m} d\left(\tilde{x}, z_{m}\right) d\left(z_{m}, x_{n+1}\right) \\
& +\alpha_{m} d^{2}\left(z_{m}, x_{n+1}\right)+\left(1-\alpha_{m}\right) d^{2}\left(z_{m}, x_{n+1}\right) \\
& +\left(1-\alpha_{m}\right) d\left(T\left(t_{m}\right) x_{n+1}, x_{n+1}\right) d\left(z_{m}, x_{n+1}\right) \\
\leq & \alpha_{m} \alpha d\left(z_{m}, \tilde{x}\right) M+\alpha_{m}\left|\overrightarrow{f(\tilde{x}) \tilde{x},} \overrightarrow{z_{m} x_{n+1}}\right\rangle+\alpha_{m} d\left(\tilde{x}, z_{m}\right) M+\alpha_{m} d^{2}\left(z_{m}, x_{n+1}\right) \\
& +\left(1-\alpha_{m}\right) d^{2}\left(z_{m}, x_{n+1}\right)+\left(1-\alpha_{m}\right) d\left(T\left(t_{m}\right) x_{n+1}, x_{n+1}\right) M \\
\leq & d^{2}\left(z_{m}, x_{n+1}\right)+\alpha_{m} \alpha d\left(z_{m}, \tilde{x}\right) M+\alpha_{m} d\left(\tilde{x}, z_{m}\right) M+d\left(T\left(t_{m}\right) x_{n+1}, x_{n+1}\right) M \\
& +\alpha_{m}\left\langle\overrightarrow{f(\tilde{x}) \tilde{x}}, \overrightarrow{z_{m} x_{n+1}}\right\rangle,
\end{aligned}
$$

where $M \geq \sup _{m, n \geq 1}\left\{d\left(z_{m}, x_{n}\right)\right\}$. This implies that

$$
\left\langle\overrightarrow{f(\tilde{x}) \tilde{x}}, \overrightarrow{x_{n+1} z_{m}}\right\rangle \leq(1+\alpha) d\left(z_{m}, \tilde{x}\right) M+\frac{d\left(T\left(t_{m}\right) x_{n+1}, x_{n+1}\right)}{\alpha_{m}} M
$$

Taking the upper limit as $n \rightarrow \infty$ first, and then $m \rightarrow \infty$, inequality (3.12) yields that

$$
\limsup _{m \rightarrow \infty} \limsup _{n \rightarrow \infty}\left\langle\overrightarrow{f(\tilde{x}) \tilde{x}}, \overrightarrow{x_{n+1} z_{m}}\right\rangle \leq 0
$$

Since

$$
\begin{aligned}
\left\langle\overrightarrow{f(\tilde{x})} \vec{x}, \overrightarrow{x_{n+1}} \overrightarrow{\tilde{x}}\right\rangle & =\left\langle\overrightarrow{f(\tilde{x}) \tilde{x}}, \overrightarrow{x_{n+1} z_{m}}\right\rangle+\left\langle\overrightarrow{f(\tilde{x}) \tilde{x}}, \overrightarrow{z_{m} \tilde{x}}\right\rangle \\
& \leq\left\langle\overrightarrow{f(\tilde{x}) \tilde{x}}, \overrightarrow{x_{n+1} z_{m}}\right\rangle+d(f(\tilde{x}), \tilde{x}) d\left(z_{m}, \tilde{x}\right) .
\end{aligned}
$$

Thus, by taking the upper limit as $n \rightarrow \infty$ first, and then $m \rightarrow \infty$ the last inequality, it follows from $z_{m} \rightarrow \tilde{x}$ and (3.13) that

$$
\limsup _{n \rightarrow \infty}\left\langle\overrightarrow{f(\tilde{x}) \tilde{x}}, \overrightarrow{x_{n+1}} \overrightarrow{\tilde{x}}\right\rangle \leq 0
$$

Finally, we prove that $x_{n} \rightarrow \tilde{x}$ as $n \rightarrow \infty$. For any $n \in \mathbb{N}$, we set $y_{n}=\alpha_{n} \tilde{x} \oplus\left(1-\alpha_{n}\right) T\left(t_{n}\right) x_{n}$. It follows from Lemma 3.1 and Lemma 3.2(i), (ii) that

$$
\begin{aligned}
& d^{2}\left(x_{n+1}, \tilde{x}\right) \leq d^{2}\left(y_{n}, \tilde{x}\right)+2\left\langle\overrightarrow{x_{n+1} y_{n}}, \overrightarrow{x_{n+1}} \vec{x}\right\rangle \\
& \leq\left(\alpha_{n} d(\tilde{x}, \tilde{x})+\left(1-\alpha_{n}\right) d\left(T\left(t_{n}\right) x_{n}, \tilde{x}\right)\right)^{2} \\
& +2\left[\alpha_{n}\left\langle\overrightarrow{f\left(x_{n}\right) y_{n}}, \overrightarrow{x_{n+1}} \overrightarrow{\tilde{x}}\right\rangle+\left(1-\alpha_{n}\right)\left\langle\overrightarrow{T\left(t_{n}\right) x_{n} y_{n}}, \overrightarrow{x_{n+1} \tilde{x}}\right\rangle\right] \\
& \leq\left(1-\alpha_{n}\right)^{2} d^{2}\left(x_{n}, \tilde{x}\right)+2\left[\alpha_{n} \alpha_{n}\left|\overrightarrow{f\left(x_{n}\right)} \overrightarrow{\tilde{x}}, \overrightarrow{x_{n+1} \tilde{x}}\right\rangle+\alpha_{n}\left(1-\alpha_{n}\right)\left\langle\overrightarrow{f\left(x_{n}\right) T\left(t_{n}\right) x_{n}}, \overrightarrow{x_{n+1}} \overrightarrow{\tilde{x}}\right\rangle\right. \\
& \left.+\left(1-\alpha_{n}\right) \alpha_{n}\left\langle\overrightarrow{T\left(t_{n}\right) x_{n} \tilde{x}}, \overrightarrow{x_{n+1}} \overrightarrow{\tilde{x}}\right\rangle+\left(1-\alpha_{n}\right)\left(1-\alpha_{n}\right)\left\langle\overrightarrow{T\left(t_{n}\right) x_{n} T\left(t_{n}\right) x_{n}}, \overrightarrow{x_{n+1}} \overrightarrow{\tilde{x}}\right\rangle\right] \\
& \leq\left(1-\alpha_{n}\right)^{2} d^{2}\left(x_{n}, \tilde{x}\right)+2\left[\alpha_{n} \alpha_{n}\left|\overrightarrow{f\left(x_{n}\right) \tilde{x}}, \overrightarrow{x_{n+1}} \overrightarrow{\tilde{x}}\right\rangle+\alpha_{n}\left(1-\alpha_{n}\right)\left\langle\overrightarrow{f\left(x_{n}\right) T\left(t_{n}\right) x_{n}}, \overrightarrow{x_{n+1}} \overrightarrow{\tilde{x}}\right\rangle\right. \\
& \left.+\left(1-\alpha_{n}\right) \alpha_{n}\left|\overrightarrow{T\left(t_{n}\right) x_{n} \tilde{x}}, \overrightarrow{x_{n+1}} \tilde{x}\right\rangle+\left(1-\alpha_{n}\right)^{2} d\left(T\left(t_{n}\right) x_{n}, T\left(t_{n}\right) x_{n}\right) d\left(x_{n+1} \tilde{x}\right)\right]
\end{aligned}
$$




$$
\begin{aligned}
& \left.=\left(1-\alpha_{n}\right)^{2} d^{2}\left(x_{n}, \tilde{x}\right)+2\left[\alpha_{n}^{2}\left|\overrightarrow{f\left(x_{n}\right) \tilde{x}}, \overrightarrow{x_{n+1}} \overrightarrow{\tilde{x}}\right\rangle+\alpha_{n}\left(1-\alpha_{n}\right) \mid \overrightarrow{f\left(x_{n}\right)} \overrightarrow{\tilde{x}}, \overrightarrow{x_{n+1}} \overrightarrow{\tilde{x}}\right)\right] \\
& =\left(1-\alpha_{n}\right)^{2} d^{2}\left(x_{n}, \tilde{x}\right)+2 \alpha_{n}\left|\overrightarrow{f\left(x_{n}\right) \tilde{x}}, \overrightarrow{x_{n+1}} \tilde{\tilde{x}}\right\rangle \\
& =\left(1-\alpha_{n}\right)^{2} d^{2}\left(x_{n}, \tilde{x}\right)+2 \alpha_{n}\left|\overrightarrow{f\left(x_{n}\right) f(\tilde{x})}, \overrightarrow{x_{n+1}} \overrightarrow{\tilde{x}}\right\rangle+2 \alpha_{n}\left|\overrightarrow{f(\tilde{x})} \overrightarrow{\tilde{x}}, \overrightarrow{x_{n+1}} \overrightarrow{\tilde{x}}\right\rangle \\
& \left.\leq\left(1-\alpha_{n}\right)^{2} d^{2}\left(x_{n}, \tilde{x}\right)+2 \alpha_{n} \alpha d\left(x_{n}, \tilde{x}\right) d\left(x_{n+1}, \tilde{x}\right)+2 \alpha_{n} \mid \overrightarrow{f(\tilde{x})} \vec{x}, \overrightarrow{x_{n+1}} \overrightarrow{\tilde{x}}\right) \\
& \leq\left(1-\alpha_{n}\right)^{2} d^{2}\left(x_{n}, \tilde{x}\right)+\alpha_{n} \alpha\left(d^{2}\left(x_{n}, \tilde{x}\right)+d^{2}\left(x_{n+1}, \tilde{x}\right)\right)+2 \alpha_{n}\left|\overrightarrow{f(\tilde{x})} \vec{x}, \overrightarrow{x_{n+1}} \overrightarrow{\tilde{x}}\right\rangle,
\end{aligned}
$$

which implies that

$$
\begin{aligned}
d^{2}\left(x_{n+1}, \tilde{x}\right) & \leq \frac{1-(2-\alpha) \alpha_{n}+\alpha_{n}^{2}}{1-\alpha \alpha_{n}} d^{2}\left(x_{n}, \tilde{x}\right)+\frac{2 \alpha_{n}}{1-\alpha \alpha_{n}}\left\langle\overrightarrow{f(\tilde{x}) \tilde{x}}, \overrightarrow{x_{n+1} \tilde{x}}\right\rangle \\
& \leq \frac{1-(2-\alpha) \alpha_{n}}{1-\alpha \alpha_{n}} d^{2}\left(x_{n}, \tilde{x}\right)+\frac{2 \alpha_{n}}{1-\alpha \alpha_{n}}\left|\overrightarrow{f(\tilde{x}) \tilde{x}}, \overrightarrow{x_{n+1}} \overrightarrow{\tilde{x}}\right\rangle+\alpha_{n}^{2} M,
\end{aligned}
$$

where $M \geq \sup _{n \geq 0}\left\{d^{2}\left(x_{n}, \tilde{x}\right)\right\}$. It then follows that

$$
d^{2}\left(x_{n+1}, \tilde{x}\right) \leq\left(1-\alpha_{n}^{\prime}\right) d^{2}\left(x_{n}, \tilde{x}\right)+\alpha_{n}^{\prime} \beta_{n}^{\prime}
$$

where

$$
\alpha_{n}^{\prime}=\frac{2(1-\alpha) \alpha_{n}}{1-\alpha \alpha_{n}} \quad \text { and } \quad \beta_{n}^{\prime}=\frac{\left(1-\alpha \alpha_{n}\right) \alpha_{n}}{2(1-\alpha)} M+\frac{1}{(1-\alpha)}\left\langle\overrightarrow{f(\tilde{x}) \tilde{x}}, \overrightarrow{x_{n+1}} \overrightarrow{\tilde{x}}\right\rangle
$$

Applying Lemma 2.8, we can conclude that $x_{n} \rightarrow \tilde{x}$. This completes the proof.

$$
\text { If } f \equiv u \text {, then the following corollary can be obtained directly from Theorem 3.5. }
$$

Corollary 3.6 Let $C$ be a closed convex subset of a complete CAT(0) space $X$, and let $\{T(t)\}$ be a one-parameter continuous semigroup of nonexpansive mappings on $C$ satisfying $\mathcal{F} \neq \varnothing$ and uniformly asymptotically regular (in short, u.a.r.) on $C$, that is, for all $h \geq 0$ and any bounded subset $B$ of $C$,

$$
\lim _{t \rightarrow \infty} \sup _{x \in B} d(T(h)(T(t) x), T(t) x)=0 .
$$

Suppose that $t_{n} \in[0, \infty), \alpha_{n} \in(0,1), x_{0} \in C$ and $\left\{x_{n}\right\}$ is given by

$$
x_{n+1}=\alpha_{n} u \oplus\left(1-\alpha_{n}\right) T\left(t_{n}\right) x_{n}, \quad \forall n \geq 0,
$$

where $\left\{\alpha_{n}\right\} \subset(0,1)$ satisfies the following conditions:

(i) $\lim _{n \rightarrow \infty} \alpha_{n}=0$;

(ii) $\sum_{n=0}^{\infty} \alpha_{n}=\infty$ and

(iii) $\lim _{n \rightarrow \infty} t_{n}=\infty$.

Then $\left\{x_{n}\right\}$ converges strongly as $n \rightarrow \infty$ to $\tilde{x}$ such that $\tilde{x}=P_{\mathcal{F}} \tilde{x}$, which is equivalent to the variational inequality (3.9). 


\section{Competing interests}

The authors declare that they have no competing interests.

\section{Authors' contributions}

Both authors read and approved the final manuscript.

\section{Acknowledgements}

The first author is supported by the Centre of Excellence in Mathematics under the Commission on Higher Education, Ministry of Education, Thailand

\section{Received: 22 December 2012 Accepted: 14 May 2013 Published: 19 June 2013}

\section{References}

1. Bruhat, F, Tits, J: Groupes réductifs sur un corps local. I. Donêes radicielles valuées. Publ. Math. IHES 41, 5-251 (1972)

2. Bridson, M, Haefliger, A: Metric Spaces of Nonpositive Curvature. Springer, Berlin (1999)

3. Brown, KS: Buildings. Springer, New York (1989)

4. Goebel, K, Reich, S: Uniform Convexity, Hyperbolic Geometry, and Nonexpansive Mappings. Series of Monographs and Textbooks in Pure and Applied Mathematics, vol. 83. Dekker, New York (1984)

5. Berg, ID, Nikolaev, IG: Quasilinearization and curvature of Alexandrov spaces. Geom. Dedic. 133, 195-218 (2008)

6. Ahmadi Kakavandi, B, Amini, M: Duality and subdifferential for convex functions on complete CAT(0) metric spaces. Nonlinear Anal. 73, 3450-3455 (2010)

7. Dehghan, H, Rooin, J: A characterization of metric projection in CAT(0) spaces. In: International Conference on Functional Equation, Geometric Functions and Applications (ICFGA 2012) 10-12th May 2012, pp. 41-43. Payame Noor University, Tabriz (2012)

8. Browder, FE: Fixed point theorems for noncompact mappings in Hilbert spaces. Proc. Natl. Acad. Sci. USA 53, 1272-1276 (1965)

9. Reich, S: Strong convergence theorems for resolvents of accretive operators in Banach spaces. J. Math. Anal. Appl. 75, 287-292 (1980)

10. Halpern, B: Fixed points of nonexpansive maps. Bull. Am. Math. Soc. 73, 957-961 (1967)

11. Shioji, N, Takahashi, W: Strong convergence theorems for asymptotically nonexpansive semi-groups in Hilbert spaces. Nonlinear Anal. 34, 87-99 (1998)

12. Suzuki, T: On strong convergence to common fixed points of nonexpansive semigroups in Hilbert spaces. Proc. Am. Math. Soc. 131, 2133-2136 (2003)

13. Song, Y, Xu, S: Strong convergence theorems for nonexpansive semigroup in Banach spaces. J. Math. Anal. Appl. 338, 152-161 (2008)

14. Dhompongsa, S, Fupinwong, W, Kaewkhao, A: Common fixed points of a nonexpansive semigroup and a convergence theorem for Mann iterations in geodesic metric spaces. Nonlinear Anal. 70, 4268-4273 (2009)

15. Cho, YJ, Ćirić, L, Wang, SH: Convergence theorems for nonexpansive semigroups in CAT(0) spaces. Nonlinear Anal. 74 6050-6059 (2011)

16. Lin, L-J, Chuang, C, Yu, ZT: Convergence theorems for some semigroups of nonexpansive mappings in complete CAT(0) spaces. Fixed Point Theory Appl. 2012, Article ID 155 (2012). doi:10.1186/1687-1812-2012-155

17. Shi, LY, Chen, RD: Strong convergence of viscosity approximation methods for nonexpansive mappings in CAT(0) spaces. J. Appl. Math. 2012, Article ID 421050 (2012). doi:10.1155/2012/421050

18. Wangkeeree, R, Preechasilp, P: Viscosity approximation methods for nonexpansive mappings in CAT(0) spaces. J. Inequal. Appl. 2013, Article ID 93 (2013). doi:10.1186/1029-242X-2013-93

19. Dhompongsa, S, Panyanak, B: On $\Delta$-convergence theorems in CAT(0) spaces. Comput. Math. Appl. 56, 2572-2579 (2008)

20. Lim, TC: Remarks on some fixed point theorems. Proc. Am. Math. Soc. 60, 179-182 (1976)

21. Kirk, WA, Panyanak, B: A concept of convergence in geodesic spaces. Nonlinear Anal. 68, 3689-3696 (2008)

22. Dhompongsa, S, Kirk, WA, Sims, B: Fixed points of uniformly Lipschitzian mappings. Nonlinear Anal. 65, 762-772 (2006)

23. Dhompongsa, S, Kirk, WA, Panyanak, B: Nonexpansive set-valued mappings in metric and Banach spaces. J. Nonlinear Convex Anal. 8, 35-45 (2007)

24. Ahmadi Kakavandi, B: Weak topologies in complete CAT(0) metric spaces, Proc. Am. Math. Soc. 141, 1029-1039 (2013) S 0002-9939(2012)11743-5

25. Xu, HK: An iterative approach to quadratic optimization. J. Optim. Theory Appl. 116, 659-678 (2003) 